\title{
O ato de cuidar em saúde mental: aspectos alinhados à cultura de segurança do paciente*
}

\section{Marcos Eduardo Pereira de Lima ${ }^{1}$ (D) https://orcid.org/0000-0003-2205-9452 Elaine Antunes Cortez ${ }^{1}$ \\ (D) https://orcid.org/0000-0003-3912-9648 \\ Viviane Lins Araújo de Almeida ${ }^{1}$ \\ (D) https://orcid.org/0000-0003-0846-9316 \\ Simone Costa da Matta Xavier ${ }^{1}$ \\ (D) https://orcid.org/0000-0003-3515-8134 \\ Fabíola Chaves Fernandes ${ }^{1}$ \\ (DiD htps://orcid.org/0000-0001-9838-1689}

Artigo extraído da dissertação de mestrado "Educação permanente em prol da segurança do paciente na saúde mental: pesquisa convergente assistencial", apresentada à Escola de Enfermagem Aurora Afonso Costa, Universidade Federal Fluminense, Niterói, RJ, Brasil.

${ }^{1}$ Universidade Federal Fluminense, Escola de Enfermagem Aurora Afonso Costa, Niterói, RJ, Brasil.
Objetivo: analisar o conhecimento sobre o cuidado prestado à pessoa que possui transtorno mental na perspectiva da segurança do paciente. Método: revisão integrativa da literatura, considerando-se a seguinte questão norteadora: "Como a Política de Segurança do Paciente pode ser incorporada na produção do cuidado às pessoas ou sujeitos em sofrimento psíquico?". Resultados: foram selecionados 12 artigos em língua inglesa nas bases de dados Scopus e MEDLINE. Predominaram os tipos de estudo revisão da literatura (42\%), estudo crítico-reflexivo (17\%) e análise de conteúdo (17\%). Conclusão: a segurança do paciente no contexto da saúde mental é mais complexa quando comparada a de outros pacientes. Observa-se maior probabilidade de eventos adversos, violências, barreiras de acesso aos serviços e tratamentos, além de uma assistência de baixa qualidade, tardia e não planejada, tendo, como principal justificativa, o processo estigmatizador. A educação permanente e o cuidado centrado na pessoa são as principais ferramentas de solução desses casos.

Descritores: Pessoas Mentalmente Doentes; Segurança do Paciente; Empatia; Saúde Mental.

\section{Como citar este artigo}

Lima MEP, Cortez EA, Almeida VLA, Xavier SCM, Fernandes FC. The act of caring in mental health: aspects aligned to patient safety culture. SMAD, Rev Eletrônica Saúde Mental Álcool Drog. 2021 abr.-jun.;17(2):92-103. doi: https://dx.doi.org/10.11606/issn.1806-6976.smad.2021.168515 


\title{
The act of caring in mental health: aspects aligned to patient safety culture
}

\begin{abstract}
Objective: to analyze the knowledge about the care given to the person who has mental disorder from the perspective of the patient's safety. Method: Integrative literature review, considering the following guiding question: "How can the Patient Safety Policy be incorporated into the production of care for people or subjects in psychic suffering?" Results: 12 English language articles were selected from the Scopus and MEDLINE databases. The types of study were: literature review $(42 \%)$, critical-reflective study (17\%) and content analysis (17\%). Conclusion: patient safety in the context of mental health is more complex when compared to other patients. There is a higher probability of adverse events, violence, barriers to access to services and treatments, as well as low quality, late and unplanned care, having as main justification the stigmatization process. Permanent education and person-centered care are the main tools for solving these cases.
\end{abstract}

Descriptors: Mentally Ill People; Patient Safety; Empathy; Mental Health.

\section{El acto de cuidar en salud mental: aspectos alineados la cultura de seguridad del paciente}

\begin{abstract}
Objetivo: analizar el conocimiento sobre el cuidado prestado à la persona que posee trastorno mental en la perspectiva de la seguridad del paciente. Método: revisión integral de la literatura, considerándose la siguiente cuestión norteadora: cómo se puede incorporar la Política de seguridad del paciente en la producción de atención para personas con problemas psicológicos? Resultados: se seleccionaron 12 artículos en inglés, en las bases de datos SCOPUS y MEDLINE. Predominaron los tipos de estudio Revisión de la literatura (42\%), Estudio crítico-reflexivo (17\%) y Análisis de contenido (17\%). Conclusión: la seguridad del paciente en el contexto de la salud mental es más compleja en comparación con otros pacientes, se observa mayor probabilidad de eventos adversos, violencias, barreras de acceso a los servicios y tratamientos, además de una asistencia de baja calidad, tardía y no planificada, teniendo como principal justificación el proceso estigmatizador. La educación permanente y el cuidado centrado en la persona son las principales herramientas de solución de estos casos.
\end{abstract}

Descriptores: Enfermos Mentales; Seguridad del Paciente; Empatía; Salud Mental. 


\section{Introdução}

Historicamente, a loucura no Brasil, a partir da chegada da Família Real no início do século XIX, sofreu ingerência com o recolhimento de pessoas apontadas como insanas na cidade do Rio de Janeiro. Essa proposta visava a avançar socialmente e economicamente e, portanto, era preciso potencializar o controle social e organizar a expansão da cidade ${ }^{(1)}$. Diante dessa conjuntura, nasce o hospital psiquiátrico, mais conhecido como manicômio, instituição esta que tinha como princípio o controle dos corpos, a remoção de pessoas com transtornos clínicos e que não tinham comportamento socialmente aceitável.

$\mathrm{O}$ atendimento prestado às pessoas nesse local era precário: viviam em condições insalubres e eram atendidas minimamente em suas necessidades básicas. O tratamento era medicalizante e, muitas vezes, era feito por contenção dos corpos como forma de disciplinar, castigar e cessar as manifestações de crises psiquiátricas.

Durante anos, a sociedade tratou a pessoa com transtorno mental como um ser "não humano", devido às ideias preconceituosas em torno da doença, o que incidiu em rejeição, asilamento e exclusão social.

Esse tipo de serviço fortaleceu-se, aumentando bruscamente ao longo dos séculos no território nacional, sendo compreendido como ordenador social. Alargou seu financiamento com o Sistema Único de Saúde (SUS), culminando em uma ampliação gigantesca de pessoas internadas até advir a Reforma Psiquiátrica Brasileira.

Essa reforma teve sua origem na Itália em 1978, a partir da Lei no 180 ou Lei da Reforma Psiquiátrica Italiana, tendo como principal responsável o psiquiatra Franco Basaglia. Esse movimento buscou transformar e indicar a reestruturação da assistência prestada pelos profissionais que atuavam no hospital psiquiátrico e a ruptura do paradigma do saber médico e do tratamento à pessoa ou sujeito em sofrimento psíquico ao longo dos anos ${ }^{(2)}$.

Essa lei e o modelo que ela orientava influenciaram o tratamento ofertado à pessoa ou sujeito em sofrimento psíquico ao redor do mundo, inclusive tendo repercussão no Brasil. Diante disso, com o avançar das políticas, da redemocratização e da reforma sanitária, o serviço prestado pelo manicômio no Brasil passou a ser questionado, o que resultou também em reivindicação dos trabalhadores por melhores condições de trabalho, bem como a qualidade do serviço oferecido às pessoas que lá estavam confinadas.

A partir desse movimento político-social, no Brasil, surge a proposta de Projeto de Lei no 3.657, do então deputado Paulo Delgado, em 1989, que visava a impulsionar a Reforma Psiquiátrica Brasileira. A Lei no 10.216 foi sancionada somente em 06 de abril de 2001 e ela aponta para a "proteção e os direitos das pessoas portadoras de transtornos mentais e redireciona o modelo assistencial em saúde mental". Esse marco reafirmou a responsabilidade do Estado para com a política de saúde mental no Brasil(3).

Com isso, iniciou-se o declínio do manicômio, surgindo novas abordagens de cuidado à pessoa que sofre transtorno mental, além de equipamentos de saúde mental estruturados pela Rede de Atenção Psicossocial (RAPS), que tem o objetivo de ser ponto de atenção para pessoas ou sujeitos em sofrimento psíquico conforme Portaria do GM no 3.088. São eles: Serviços de Residências Terapêuticas (SRT), moradia destinada à pessoa que permaneceu por longo período de internação dentro do hospital psiquiátrico e que perdeu laço familiar; Centro de Atenção Psicossocial (CAPS), serviço de base territorial do usuário composto por equipe multidisciplinar e que tem a missão de ser um ponto de atenção à saúde da pessoa com transtorno mental, bem como atender às situações de crise, entre outros ${ }^{(4)}$.

O conceito de cuidado em saúde mental está alinhado aos princípios do SUS e à Lei no 10.216, na universalidade do acesso aos melhores serviços em qualquer nível de atenção, de modo inclusivo e comunitário; na integralidade de ações, sendo possível avaliar o todo sem distinção e preconceito em ambiente terapêutico; na equidade de direitos e ofertas de serviço com humanidade; na descentralização políticoadministrativa, envolvendo e compartilhando, com outros atores, os processos decisórios para melhor desenvolvimento das ações estratégicas e o controle social, com a participação efetiva da sociedade civil, instituições, entre outros ${ }^{(3,5)}$.

$\mathrm{Na}$ composição desse cuidado, incluem-se a rede de atenção à saúde mental, o território e a autonomia da pessoa ou sujeito com sofrimento psíquico. Essa rede comunitária no campo da saúde mental é fundamental para o cuidado em saúde na perspectiva da reforma psiquiátrica, pois compõe serviços que estarão aptos para o acolhimento das demandas, "que pode garantir resolutividade, promoção da autonomia e da cidadania das pessoas com transtornos mentais"(5). Quanto ao território, o conceito não está localizado apenas na área geográfica, mas, sim, baseado nas relações constituídas na comunidade, elevando "saberes e potencialidades".

Para tanto, o conceito de cuidado da pessoa ou sujeito em sofrimento psíquico deve estar atrelado à moradia digna, à oportunidade de ofício, à assistência farmacêutica, à redução do estigma a partir da educação coletiva na comunidade, à garantia de acesso aos serviços além dos de saúde e à (re) avaliação das leis e políticas públicas que impactam significativamente o campo da saúde mental(6). 
Atualmente, na lógica da qualidade da produção do cuidado, o Ministério da Saúde, junto à Organização Mundial da Saúde (OMS), vem incentivando instituições de saúde a aderirem à Política de Segurança do Paciente. O pai da Medicina, Hipócrates (460 a 370 a.C.), já pensava que o cuidado poderia trazer algum tipo de dano(7), hoje conhecido como evento adverso, dano decorrendo do cuidado prestado.

A Portaria do Ministério da Saúde no 529, de $1^{\circ}$ de abril de 2013, instituiu o Programa Nacional de Segurança do Paciente em todo território nacional, tendo como premissa contribuir para a melhoria da assistência dos estabelecimentos de saúde, sejam serviços públicos ou privados. Além disso, reforçou a Lei Orgânica da Saúde, onde se destaca a responsabilidade das esferas federais, municipais e estaduais para a elaboração das normas e atividades dos serviços de saúde e estabelece o SUS a fim de coordenar e definir o sistema de vigilância sanitária; controlar, fiscalizar procedimentos e produtos, bem como avaliar as ações e serviços ${ }^{(8)}$.

A segurança do paciente é uma prioridade assumida com OMS. Logo, o Brasil deve incentivar o conhecimento multidisciplinar, contribuindo, assim, com a qualificação do cuidado, a integração dos conselhos da classe trabalhadora, as instituições de ensino e pesquisa e o apoio aos gestores das unidades de saúde em todo território nacional. Assim, constituiu-se o Programa Nacional de Segurança do Paciente (PNSP) com o objetivo de criar os núcleos de segurança do paciente, além de incentivar as atividades educativas em cada serviço de saúde; aproximar pacientes e familiares para a discussão; disseminar informações sobre a segurança do paciente na comunidade e introduzir a temática nas instituições de ensino técnico e superior (graduação e pós-graduação) $)^{(8)}$.

O PNSP não é a solução para todos os entraves de um serviço, mas ele poderá, sim, impulsionar articulações externas e internas em conjunto com outras políticas públicas de saúde em prol da qualidade da assistência, como, por exemplo, a (re) organização da rede de cuidado, dos serviços de atenção primária à terciária; o incentivo ao profissional como protagonista no papel que desempenha e o reforço do compromisso dos gestores das unidades de saúde e seus representantes públicos ${ }^{(8)}$.

A relevância deste estudo possibilita uma reflexão de como é produzido o cuidado às pessoas ou sujeitos em sofrimento psíquico e como essa nova política pode ser aplicada no cotidiano dos serviços de saúde mental. Somado a isso, contribuir para a potencialização e a discussão dos processos de trabalho seguro dos profissionais de saúde na perspectiva da saúde mental, bem como para os estudantes que tenham interesse no campo da Psiquiatria. Afinal, é preciso qualificar futuros profissionais para exercerem atividades responsáveis e seguranças no âmbito dos serviços de saúde. Com isso, é possível reconhecer muitas pesquisas voltadas para a história da Psiquiatria, bem como a Reforma Psiquiátrica. Mas há lacunas do conhecimento sobre a área da saúde mental e a segurança do paciente, pois, além de ser uma política recente, a maioria dos estudos publicados relacionou as temáticas, focando na gestão de riscos, protocolos de segurança do paciente e na farmacologia, não permitindo refletir sobre o cuidar da pessoa com sofrimento psíquico e suas peculiaridades, não esquecendo que "à medida que a efetividade do cuidado de saúde aumenta, cada vez mais pacientes envelhecem mantendo suas doenças sob controle"(9). Desse modo, objetiva analisar o conhecimento sobre o cuidado prestado à pessoa que possui transtorno mental na perspectiva da segurança do paciente, a partir de uma revisão de literatura com uma análise reflexiva dos achados.

\section{Método}

Trata-se de uma revisão de narrativa da literatura internacional a partir da formulação da questão norteadora, com busca em bases de dados on-line, seguida de categorização dos achados, avaliação dos estudos incluídos, discussão, com análise e interpretações mais amplas dos resultados ${ }^{(10)}$. Considerou-se como questão norteadora: "Como a Política de Segurança do Paciente pode ser incorporada à produção do cuidado às pessoas ou sujeitos em sofrimento psíquico?".

Para a definição dos descritores, foi utilizada a estratégia $\mathrm{PICO}^{(11)}$ em que $\mathrm{P}$ significa o paciente ou problema e pode-se descrever um único paciente, um grupo de pacientes com uma condição particular ou um problema de saúde. Neste estudo, há referência às pessoas ou sujeitos em sofrimento psíquico. I trata da intervenção proposta, que pode incluir uma exposição, ser terapêutica, preventiva, diagnóstica, prognóstica, administrativa ou relacionada a assuntos econômicos. Foi considerada a inserção da Política de Segurança do Paciente na produção do cuidado às pessoas mentalmente doentes. C constitui controle ou comparação, contudo, não se empregará este elemento. O é o desfecho, significando o resultado esperado, que, neste caso, é identificar como a Política de Segurança do Paciente pode ser incorporada na produção do cuidado às pessoas ou sujeitos em sofrimento psíquico, delimitando, assim, o tema de estudo.

A partir disso, as palavras foram referenciadas nos Descritores em Ciências da Saúde (DeCS) e os termos Medical Subject Headings (MESH) nas três bases eletrônicas de dados Culmulative Index to Nursing and Allied Health (CINAHL), Medical Literature Analysis and Retrieval System Online (MEDLINE via PubMed) e Medical Subject Headings (Scopus), conforme a Figura 1 a seguir. 


\begin{tabular}{|c|c|c|c|c|}
\hline PICO Estratégia* & Descritores $\mathrm{DeCS}^{\dagger}$ & Sinônimos & Descritores $\mathrm{MESH}^{\ddagger \dagger}$ & Entrytems \\
\hline $\begin{array}{l}\text { Pessoas } \\
\text { Mentalmente } \\
\text { Doentes }\end{array}$ & $\begin{array}{l}\text { Pessoas } \\
\text { Mentalmente } \\
\text { Doentes }\end{array}$ & $\begin{array}{l}\text { Doentes Mentais; } \\
\text { Pacientes Mentais. }\end{array}$ & $\begin{array}{l}\text { Mentally III Person } \\
\text { Person, Mentally III } \\
\text { Persons, Mentally III } \\
\text { Mentally III } \\
\text { III, Mentally } \\
\text { Mental Patients }\end{array}$ & Mentally III Persons \\
\hline $\begin{array}{l}\text { Segurança } \\
\text { do Paciente }\end{array}$ & $\begin{array}{l}\text { Segurança } \\
\text { do Paciente }\end{array}$ & Nenhum & Patient Safety & Patient Safety \\
\hline Cuidado & Empatia & $\begin{array}{l}\text { Benevolência; Complacência } \\
\text { (Sentimento); Condescendência; } \\
\text { Compaixão; Consideração; } \\
\text { Cuidado; Cuidados; Cuidar; } \\
\text { Deferência. }\end{array}$ & $\begin{array}{l}\text { - Caring } \\
\text { - Compassion }\end{array}$ & Empathy \\
\hline
\end{tabular}

*PICO Estratégia; ${ }^{\star}$ DeCS = Descritores em Ciências da Saúde; ${ }^{\ddagger}$ MESH = Medical Subject Readings

Figura 1 - Definição dos descritores. Rio de Janeiro, RJ, Brasil, 2018

Para todas as buscas nas bases de dados, foi utilizada a estratégia dos operadores booleanos and e or, permitindo realizar associações.

Foram estabelecidos os seguintes critérios de inclusão: artigos a partir de 2013 até 2019, em língua portuguesa, inglesa ou espanhola. Definiram-se como critérios de exclusão: artigos duplicados e artigos que não atenderam à pergunta da pesquisa. A coleta de dados ocorreu no período de junho 2018 a janeiro de 2019. Foram identificados 132 estudos nas respectivas bases de dados conforme a Figura 2.

\begin{tabular}{|lc|}
\hline Bases de dados & $\mathbf{N}^{\circ}$ de estudos $(\mathbf{n}=\mathbf{1 3 2})$ \\
\hline CINAHL $^{*}$ & 12 \\
MEDLINE $^{\dagger}$ & 52 \\
Scopus $^{\ddagger}$ & 68 \\
\hline
\end{tabular}

${ }^{*} \mathrm{CINAHL}=$ Cumulative Index to Nursing and Allied Health Literature; ${ }^{\dagger}$ MEDLINE = Medical Literature Analysis and Retrieval System Online; ${ }^{\ddagger}$ Scopus = SciVerse Scopus

Figura 2 - Números de estudos nas bases de dados $(n=132)$ incluídos na revisão integrativa. Rio de Janeiro, Brasil, 2019
$\mathrm{Na}$ base de dados CINAHL, foram encontrados doze estudos; sete artigos foram descartados após a leitura dos resumos e cinco estudos selecionados para a leitura na íntegra. Na MEDLINE via PubMed, foram encontrados 52 estudos e 41 artigos foram eliminados após a leitura dos resumos, havendo um artigo duplicado, o que resultou em dez estudos para a leitura na íntegra. Na base de dados Scopus, foram encontrados 68 estudos; destes, 39 foram eliminados após a leitura dos resumos e 29 estudos separados para a leitura na íntegra.

$\mathrm{Na}$ Figura 3, no fluxograma conforme recomenda o PRISMA, apresentam-se os dados da exclusão. 


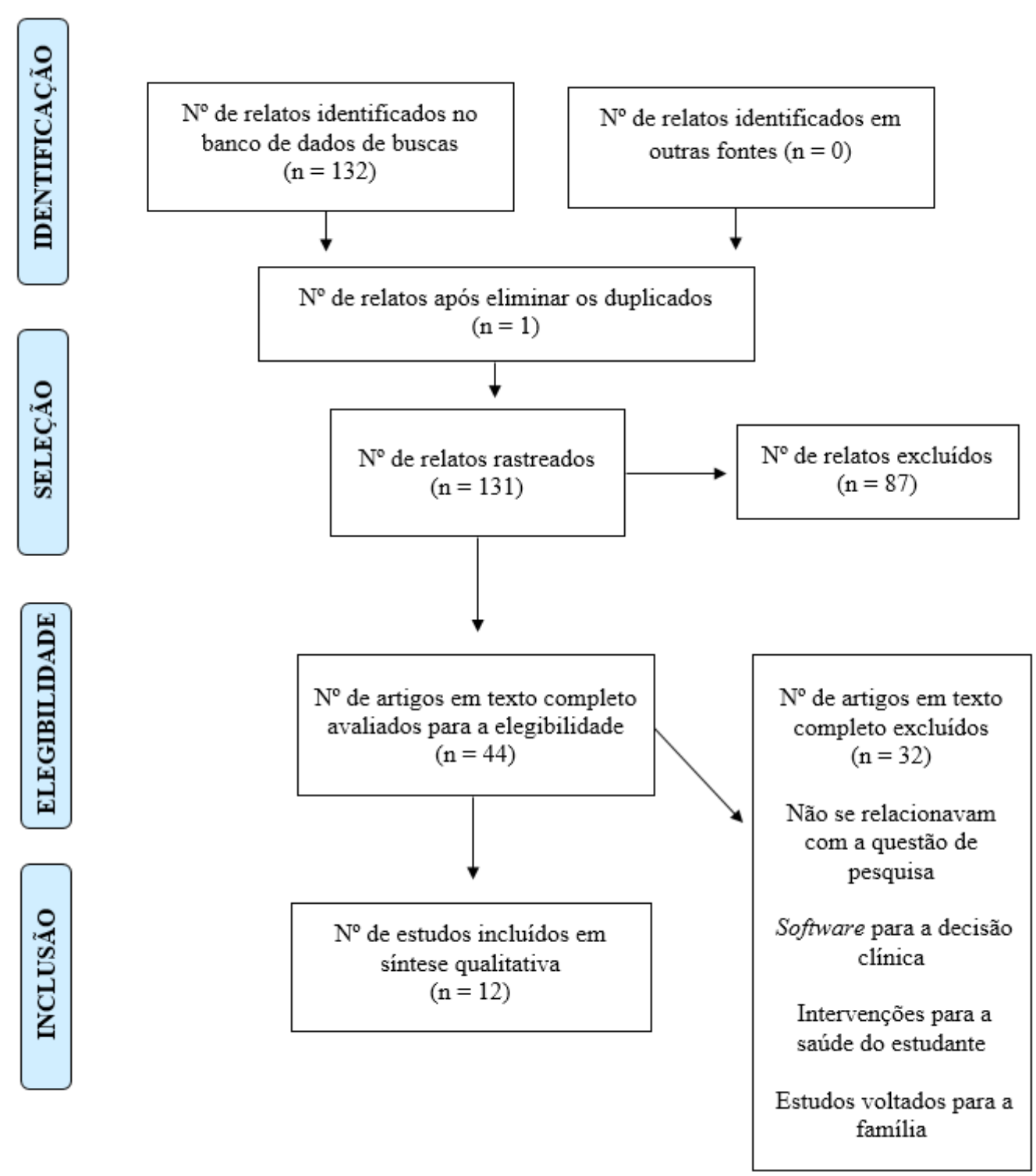

Figura 3 - Fluxograma, segundo o Prisma, para a seleção dos estudos encontrados nas bases de dados. Rio de Janeiro, Brasil, 2019

\section{Resultados}

A amostra final constituiu-se de 12 artigos, sendo extraídas as seguintes sínteses, conforme a Figura 4: autor; título; periódico; ano de publicação; tipo de estudo; origem; base de dados e Qualis da revista científica. Foram levados em consideração a autenticidade, os aspectos éticos e a autoria dos estudos publicados.

\begin{tabular}{|c|c|c|c|c|c|}
\hline $\mathbf{N}^{\circ}$ & Autores, título, periódico e ano de publicação & Tipo de estudo & Origem & Base & Qualis \\
\hline 01 & $\begin{array}{l}\text { Johnston D. What makes a difference to patients? } \\
\text { IntRevPsychiatry } 2013^{(5)} \text {. }\end{array}$ & Crítico e reflexivo & EUA $^{*}$ & Scopus $^{\dagger}$ & B1 \\
\hline 02 & $\begin{array}{l}\text { Kowalski SL, Anthony M. Nursing's evolving role in patient } \\
\text { safety. Am J Nurs } 2017^{(6)} \text {. }\end{array}$ & Análise de conteúdo & $E \cup A^{*}$ & Scopus $^{\dagger}$ & A1 \\
\hline 03 & $\begin{array}{l}\text { Johnson J, Hall LH, Berzins K, Baker J, Melling K, } \\
\text { Thompson C. Mental healthcare staff well-being and } \\
\text { burnout: A narrative review of trends, causes, implications, } \\
\text { and recommendations for future interventions. Int J Ment } \\
\text { Health Nurs } 2018^{(7)} \text {. }\end{array}$ & Revisão de literatura & $E \cup A^{*}$ & Scopus $^{\dagger}$ & B2 \\
\hline
\end{tabular}




\begin{tabular}{|c|c|c|c|c|c|}
\hline $\mathbf{N}^{\circ}$ & Autores, título, periódico e ano de publicação & Tipo de estudo & Origem & Base & Qualis \\
\hline 04 & $\begin{array}{l}\text { Hannigan B, Simpson A, Coffey M, Barlow S, Jones A. } \\
\text { Care coordination as imagined, care coordination as done: } \\
\text { Findings from a cross-national mental health systems study. } \\
\text { Int J IntegrCare } 2018^{(8)} \text {. }\end{array}$ & $\begin{array}{l}\text { Abordagem } \\
\text { qualitativa }\end{array}$ & Inglaterra & Scopus $^{\dagger}$ & B1 \\
\hline 05 & $\begin{array}{l}\text { Forouzan AS, Ghazinour M, Dejman M, Rafeiey H, } \\
\text { Baradaran Eftekhari M, San Sebastian M. Service users } \\
\text { and providers expectations of mental health care in Iran: A } \\
\text { qualitative study. Iran J Public Health } 2013^{(9)} \text {. }\end{array}$ & Análise de conteúdo & Irã & Scopus $^{\dagger}$ & B1 \\
\hline 06 & $\begin{array}{l}\text { Polacek MJ, Allen DE, Damin-Moss RS, Schwartz AJA, } \\
\text { Sharp D, Shattell M, et al. Engagement as an Element } \\
\text { of Safe Inpatient Psychiatric Environments. J Am } \\
\text { PsychiatrNursAssoc } 2015^{(10)} \text {. }\end{array}$ & Revisão de literatura & $E^{*} A^{*}$ & Scopus $^{\dagger}$ & A1 \\
\hline 07 & $\begin{array}{l}\text { Grant C, Ballard ED, Olson-Madden JH. An Empowerment } \\
\text { Approach to Family Caregiver Involvement in Suicide } \\
\text { Prevention: Implications for Practice. Fam J } 2015^{(11)} \text {. }\end{array}$ & Revisão de literatura & $E^{\prime} A^{*}$ & Scopus $^{\dagger}$ & B1 \\
\hline 08 & $\begin{array}{l}\text { Ashmore T, Spangaro J, Mcnamara L. 'I was raped by } \\
\text { Santa Claus': Responding to disclosures of sexual assault } \\
\text { in mental health inpatient facilities. Int J Ment Health Nurs } \\
2015^{(12)} \text {. }\end{array}$ & $\begin{array}{l}\text { Exploratório e } \\
\text { descritivo }\end{array}$ & $E \cup A^{*}$ & Scopus $^{\dagger}$ & B2 \\
\hline 09 & $\begin{array}{l}\text { Slemon A, Jenkins E, Bungay V. Safety in psychiatric } \\
\text { inpatient care: The impact of risk management culture on } \\
\text { mental health nursing practice. NursInq } 2017^{(13)} \text {. }\end{array}$ & Crítico e reflexivo & Canadá & Scopus $^{\dagger}$ & A1 \\
\hline 10 & $\begin{array}{l}\text { Reeves } E \text {, Henshall C, Hutchinson M, Jackson D. Safety of } \\
\text { service users with severe mental illness receiving inpatient } \\
\text { care on medical and surgical wards: A systematic review. Int } \\
J \text { Ment Health Nurs } 2018^{(14)} \text {. }\end{array}$ & Revisão da literatura & $E \cup A^{*}$ & Scopus $^{\dagger}$ & B2 \\
\hline 11 & $\begin{array}{l}\text { Sandra M, Scott BP, Heather S, Bonnie K, Joy CM. } \\
\text { Beyond silence: protocol for a randomized parallel-group } \\
\text { trial comparing two approaches to workplace mental } \\
\text { health education for healthcare employees. BMC Medical } \\
\text { Education } 2015^{(15)} \text {. }\end{array}$ & Estudo randomizado & Canadá & MEDLINE ${ }^{\ddagger}$ & $\mathrm{A} 1$ \\
\hline 12 & $\begin{array}{l}\text { Margaret O, Conal W, Sean H. Violence within mental } \\
\text { health services: how to enhance risk management. Risk } \\
\text { Manag Healthc Policy, } 2018^{(16)} \text {. }\end{array}$ & Revisão da literatura & Irlanda & MEDLINE $^{\ddagger}$ & $\mathrm{A} 1$ \\
\hline
\end{tabular}

*EUA = Estados Unidos da América; ${ }^{+}$Scopus = SciVerse Scopus; ${ }^{\ddagger}$ MEDLINE = Medical Literature Analysis and Retrieval System Online.

Figura 4 - Síntese das publicações selecionadas quanto ao autor, título, periódico e ano de publicação, tipo de estudo, origem, base de dados e Qualis da revista. Rio de Janeiro, Brasil, 2019

Os 12 artigos selecionados foram publicados na língua inglesa. A maioria é proveniente dos EUA $(58,3 \%)$ e as demais publicações tiveram sua origem no Canadá $(16,6 \%)$, Inglaterra (8,3\%), Irã $(8,3 \%)$ e Irlanda $(8,3 \%)$. De acordo com as bases de dados pesquisadas, $83 \%$ dos artigos estão localizados na base de dados Scopus e $17 \%$ na base de dados MEDLINE. Quanto ao tipo de estudo, predominaram os tipos revisão da literatura (42\%), estudo crítico-reflexivo (17\%) e análise de conteúdo (17\%); em menor frequência, surgem os estudos randomizados $(8 \%)$, exploratório e descritivo (1\%) e abordagem qualitativa (1\%).

\section{Discussão}

Conforme os critérios estabelecidos para a seleção dos artigos, um dado relevante é que exclusivamente foram localizados artigos em língua inglesa, fato este que comprova a importância da divulgação científica do estudo sobre a segurança do paciente e sua interface com a saúde mental, inclusive, no Brasil.

Os artigos selecionados, após a leitura e a análise integral de seus conteúdos, foram categorizados em quatro eixos temáticos, objetivando ampliar as discussões e reflexões sobre o tema proposto.

Categoria um: histórico da segurança do paciente psiquiátrico nos serviços de saúde (12-13,20). $^{2}$

A evolução do papel da Enfermagem na segurança do paciente, até 1919, era voltada para a assepsia e a nova teoria dos germes. À medida que o atendimento ao paciente se tornou algo mais complexo, novas medidas foram incorporadas e uma visão mais holística sobre foi adotada. Em 1930, surgem o conceito de prevenção de erros e as primeiras padronizações de cuidado. Esses marcos foram precursores das ideias progressistas da década de 1950, quando foram criados diferentes níveis de atenção de acordo com a complexidade do paciente (intensivo, intermediário etc.). Nas décadas de 1960 e 1970, foram reconhecidas as fontes de infecções hospitalares e iniciados os programas de prevenção. Por fim, no período de 2000 a 2015, o desempenho humano passou a ser analisado como causa dos cuidados de saúde frente a fatores sistêmicos ${ }^{(13)}$. 
Na Psiquiatria, a segurança do paciente já era discutida mundialmente no final da década de 1970, culminando na Reforma Psiquiátrica e no Movimento da Luta Antimanicomial no Brasil a partir dos manifestos dos trabalhadores, familiares, usuários e associações. Com a legenda "por uma sociedade sem manicômios", já pleiteavam a supressão dos manicômios, mudanças nas práticas assistenciais e das políticas públicas, o uso racional de medicamentos, com o planejamento para o alívio do sofrimento, e a não mercantilização da loucura(24).

No que tange à discussão da segurança do paciente internacionalmente, em 1998, o Institute of Medicine (IOM) estabeleceu o Comitê de Qualidade da Saúde na América a fim de proporcionar melhorias nos cuidados de saúde, inclusive, no campo da saúde mental. Percebeu-se que muitos pacientes desenvolviam ou agravavam doenças crônicas em longo prazo devido às farmacoterapias aplicadas no tratamento de doenças psiquiátricas ${ }^{(12)}$.

A gestão de cuidados passou a ir além do tradicional hospital e abordar o estilo de vida do paciente, adotando o aconselhamento como estratégia terapêutica. Os pacientes também se tornaram mais conscientes de seu diagnóstico e registros, o que auxiliava na prevenção de erros médicos e na adesão ao tratamento(12).

Esses apontamentos robustecem a relevância dos movimentos da Reforma Psiquiátrica. Apesar da pauta não trazer o termo "segurança do paciente" como destaque, ela já denunciava diversas iatrogenias que o hospital psiquiátrico produzia, como o uso desregrado de medicações, o tratamento como castigo, o modelo clássico da Medicina como sendo a única fonte de atenção à saúde, a exclusão social da pessoa ou sujeito com transtorno mental, a violação de direitos humanos, entre outros.

A Enfermagem possui diversas práticas de segurança para a proteção do paciente contra eventos adversos no cuidado. A discussão da segurança do paciente dentro da saúde mental ainda é limitada e, constantemente, substituída pela noção de risco do paciente que, na verdade, se traduz nos danos criados por ele, como o suicídio e as manifestações de violência(20).

Observa-se que, historicamente, as questões ligadas à segurança do paciente eram atribuídas a fatores não humanos, como microrganismos patogênicos. Com o tempo, houve uma mudança de paradigma, acrescentando que os fatores humanos também representam impacto importante na segurança do paciente. Como exemplo disto, descrevem-se os erros de pessoal, as falhas técnicas de equipamento e a ausência de educação permanente.

No contexto da saúde mental, a grande quantidade de medicamentos ministrados aumenta a possibilidade de erros, principalmente no setor de Enfermagem, devido à sua proximidade com o paciente. Além disso, os estigmas a que estão submetidos os pacientes psiquiátricos contribuem com a institucionalização, gerando um processo de racionalização de práticas nocivas sob a justificativa de manutenção da segurança, dando espaço a negligências no cuidado(20).

A Política Nacional de Educação Permanente em Saúde (PNEPS) surgiu como estratégia do Ministério da Saúde para o fortalecimento do SUS, em 13 de fevereiro de 2004, com a Portaria no $198^{(25)}$. Ela tem a premissa de desenvolver a prática reflexiva dos profissionais do campo saúde, promovendo o aprendizado coletivo significativo, corroborando a Política de Segurança do Paciente(26).

Nesse sentido, a educação permanente é uma ferramenta potente na garantia da segurança do paciente por promover o aprendizado organizacional ao analisar os processos e as ações do cotidiano, permitindo, também, que o trabalhador seja um protagonista competente e problematizador do ato de cuidar.

\section{Categoria dois: impactos da saúde mental dos profissionais} frente à segurança do paciente psiquiátrico ${ }^{(14-22)}$.

Em consonância com a abordagem sobre os fatores humanos mencionada na categoria um, a categoria dois destina-se a apresentar um retrato da saúde mental dos profissionais da Psiquiatria e como este pode impactar a segurança dos pacientes psiquiátricos.

O absenteísmo é maior em profissionais da área de saúde do que nos de outros setores, o que gera custos para as organizações. Tais custos podem ser ainda mais elevados quando o trabalhador permanece em serviço apesar de sua condição, podendo resultar em um trabalho de má qualidade, implicando erros e acidentes ${ }^{(22)}$. 0 Burnout - estado de esgotamento mental diante da vida profissional - frequentemente é associado a quedas na qualidade do atendimento e na segurança do paciente, aumentando a taxa de absenteísmo e de rotatividade de pessoal ${ }^{(14)}$.

Entre as causas de estresse e esgotamento mental das equipes de saúde, destacam-se a carga de trabalho excessiva, a falta de oportunidade para desenvolver habilidades, a falta de redes de apoio, a liderança fraca, a falta de capacitação de pessoal e o subfinanciamento dos serviços de saúde mental, além do trabalho emocional para cuidar de pacientes mentalmente doentes e dos altos níveis de violência(14).

Conforme prevê a Lei no 10.216, a internação psiquiátrica pode ocorrer de três formas: primeiro voluntária, com o consentimento do usuário; segundo - involuntária, aquela que se dá sem o consentimento do usuário e a pedido de terceiro e a terceira - internação compulsória, determinada pela Justiça( ${ }^{(3)}$.

Ressalta-se que a internação involuntária de pacientes com transtornos mentais é exaustiva, tanto para os pacientes quanto para seus cuidadores, pois alguns procedimentos, como a medicação forçada, a contenção, a reclusão e as medidas coercitivas, são considerados de 
alto risco, impactando a segurança do paciente e a saúde do profissional cuidador.

O maior registro de exaustão emocional é na equipe de Enfermagem ${ }^{(14)}$. Os trabalhadores com problemas de saúde mental geralmente não se reconhecem adoecidos ou sofrem em silêncio. Os colegas de trabalho ficam sem saber como ajudar e, em algumas ocasiões, são estigmatizados. Nesse sentido, propõem-se ações educativas como o aconselhamento em grupos de autoajuda, as discussões em grupos sobre estigma, depressão e educação em saúde mental, além de acompanhamento psicológico para o auxílio de funcionários com problemas de saúde mental no contexto do trabalho em saúde ${ }^{(22)}$. O reconhecimento profissional, aliado a uma gestão participativa e democrática, também pode contribuir no enfrentamento dessa problemática.

Categoria três: coordenação do cuidado em saúde mental para a segurança do paciente.

A categoria terceira evidencia a coordenação do cuidado em saúde mental como destinada a "garantir que as necessidades sejam atendidas e serviços integrados sejam fornecidos", o que, em prática, corrobora a segurança do paciente ${ }^{(15)}$. Por exemplo, a necessidade de acolhimento temporário devido à crise, à tentativa de suicídio, às demandas clínicas e odontológicas, à vulnerabilidade social e territorial, à reavaliação de seu projeto terapêutico, ao espaço de promoção terapêutica, à oferta de medicações que favoreçam o equilíbrio, ao acesso à cultura e arte, entre outros. Essa afirmativa vai ao encontro da seriedade do mandato da Rede de Atenção Psicossocial quanto à organização assistencial do cuidado em que cada dispositivo (CAPS, SRT, Hospital Geral, Unidade de Urgência e Emergência, Unidade de Acolhimento, Ambulatório, entre outros) tem a responsabilidade de ofertar, de forma segura, singular e responsável, o melhor tratamento.

Falar de coordenação do cuidado traduz a discussão sobre a continuidade e o gerenciamento de casos. A saúde mental coloca a complexidade do paciente em sofrimento psíquico em evidência, envolvendo questões psicossociais e biológicas, que somente podem ser trabalhadas com sucesso diante do trabalho em equipe e de uma abordagem multiprofissional e, para tanto, o gerenciamento dos casos é uma ferramenta indispensável. Coordenar o cuidado em saúde mental também impõe desafios, esbarrando em questões administrativas e burocráticas que dificultam o acesso aos serviços, além de uma preocupação excessiva dos gestores com o desempenho, distorcendo o objetivo principal de se coordenar o cuidado. Para além desses obstáculos, os recursos escassos generalizam as atribuições e os profissionais que coordenam o cuidado demonstram preocupação com a qualidade da relação usuário-serviço-profissional, bem como com o processo de adaptação entre estes ${ }^{(15)}$.

Estudos apontaram que o engajamento construído entre enfermeiros e pacientes, no campo da saúde mental, como um fator protetor, que vem a somar-se à segurança do paciente ${ }^{(15,17)}$. Entende-se por engajamento "estar clinicamente envolvido com um paciente enquanto o paciente se move em direção aos objetivos do tratamento clínico" ou ainda, em uma visão organizacional envolvimento dos enfermeiros com seus ambientes de trabalho. Em ambientes institucionalizados, o engajamento é descrito pelo Instituto para Ambientes Seguros (ISE) da American Psychiatric Nurses Association (APNA) como sendo um fator que afeta a segurança dos ambientes de internação.

O cuidado deve ser permeado de atos que visam assegurar a coordenação do cuidado, tendo uma visão mais ampla da prática, bem como, é preciso implementar políticas de gestão do cuidado para movimentar a clínica e desenvolver práticas centradas na pessoa(9).

De modo geral, o engajamento possibilita uma melhor conexão e comunicação com o paciente, uma relação terapêutica positiva, que antecipa planos de cuidados mais assertivos, impactando positivamente na adesão ao tratamento. Do ponto de vista organizacional, engajar-se no trabalho em Enfermagem está intimamente ligado ao reconhecimento, à realização de experiências gratificantes, aumentando o senso de eficácia profissional. Como fatores que podem vir a dificultar o engajamento, descrevem-se: a falta de treinamento, supervisão e confiança, além do estigma, ameaças de violência e processo de negação do diagnóstico psiquiátrico(17).

Categoria quatro: Fatores que implicam na segurança do paciente em sofrimento psíquico ${ }^{(16-19,21)}$

Diferentes fatores podem impactar decisivamente na segurança do paciente em sofrimento psíquico(16). As longas distâncias dos serviços, geralmente localizadas em centros urbanos, dificultam o acesso das populações mais afastadas, bem como, o tempo de espera por uma vaga em consulta. As informações sobre saúde também são dificultadas, já que há predomínio de uma linguagem técnica, de difícil entendimento, impactando na adesão das práticas de promoção de saúde. Outra problemática envolvendo a acessibilidade é a escassez de serviços domiciliares, de acompanhamento pós-crise e centros comunitários.

A qualidade das relações interpessoais também é apontada como um fator de impacto na segurança do paciente. Relações construídas tendo o respeito e a empatia como base, de formas não estigmatizadoras, tendem a influenciar em desfechos positivos, preservando a individualidade dos sujeitos dentro de uma perspectiva humanista. A participação nos processos decisórios, 
dentro de um ambiente terapêutico é importante para manter uma relação de confiança, preservando a autonomia dos sujeitos. Destaca-se ainda a importância da adequação cultural dentro do processo de cuidar. Em contextos de vulnerabilidade, os profissionais de saúde devem considerar aspectos sociais, incluindo a formação do usuário para que possam ser desenvolvidas ações terapêuticas efetivas(16).

Importante destacar que há pacientes que são prejudicados diante da assistência ofertada. Assim, o cuidado à pessoa vulnerável, torna-se um desafio. É imprescindível compreender que o cuidado seguro, não está só em seguir protocolos. Ele deverá ser considerado primeiramente como um comportamento/ conhecimento a ser adotado e adaptado a cada caso, pois entende-se que toda pessoa se apresenta de forma singular(9).

Contrapondo esta problemática com à rede de atenção psicossocial brasileira, reafirma-se a importância de se trabalhar pela lógica da longitudinalidade e regionalização dos serviços, resgatando os princípios de integralidade do cuidado e atributos como a competência cultural, que regem os serviços de atenção do sistema único de saúde.

A abordagem familiar é um importante laço na produção do cuidado compartilhado, envolvendo profissionais e cuidadores ${ }^{(18)}$. Como exemplificam os autores, diante de situações específicas, como a de risco de suicídio, disponibilizar conhecimentos educativos às famílias diminui a sobrecarga do cuidador, fornecendo informações sobre o manejo de situações de risco e reconhecendo os sinais de alerta que indicam quando procurar auxílio profissional. $\mathrm{O}$ estudo sugeriu um modelo de educação familiar denominado COPE, que consiste na educação em saúde realizada por um profissional utilizando materiais didáticos que exemplificam casos e agem no empoderamento familiar, direcionando os cuidadores a se prepararem antes de uma determinada situação ocorrer.

O fator "risco de violência sexual em pacientes psiquiátricos" é compreendido como preocupante dentro do contexto de um ambiente de internação segura. Por meio da apresentação de diferentes casos, os autores explicitaram as complexidades do cuidar de indivíduos que podem ser incapazes de fornecer relatos coerentes de agressões, podendo, muitas vezes, revivenciá-las diante de gatilhos atuais ou mesmo relatar experiências passadas $^{(19)}$. Diante disso, cabe ao profissional estar capacitado para abordar e identificar as diferenças dos relatos, garantindo que intervenções desnecessárias não sejam realizadas ou mesmo não deixando de executar as que necessitam de ação.

Alguns fatores podem dificultar o manejo desses casos pelos profissionais, como a cultura do medo e o rigor disciplinar presentes em ambientes institucionais, podendo atuar como silenciadores das denúncias, mesmo quando o profissional as identifica. Quando o agressor é membro da própria equipe, as implicações sobre a confiabilidade do relato são postas em dúvida diante da psicose ${ }^{(19)}$

Ainda diante de um contexto de internação, analisa-se como se dá a internação de pacientes com diagnóstico psiquiátrico em clínicas médicas e cirúrgicas ${ }^{(21)}$. Entende o sujeito em sofrimento psíquico, desprendendo-se do conceito da Psiquiatria tradicional, como um indivíduo único, particular e que, para além de uma comorbidade psiquiátrica, pode vir a apresentar outras necessidades de saúde de origem clínica.

A probabilidade de um paciente que sofre de transtornos mentais apresentar efeitos adversos e complicações durante a internação é maior do que na população em geral, como, por exemplo, úlceras e fraturas no pós-operatório(21). Justifica-se esse achado pela redução dos cuidados prestados aos pacientes, muitas vezes, não planejados ou omissos, aumentando o tempo de internação e da terapêutica medicamentosa, resultando em custos de atendimentos hospitalares ainda maiores.

A gravidade da doença mental aumenta a probabilidade de dano ou morte, em especial, a esquizofrenia, que se apresenta com maiores chances de efeitos adversos do que quando comparada a outros diagnósticos de saúde mental. Em uma visão geral, os pacientes psiquiátricos, quando internados para a realização de algum tratamento hospitalar, recebem cuidados de baixa qualidade, sendo importante planejar uma assistência sistematizada, que supere os estigmas e as desigualdades enfrentadas por essa população. Fortalecer as ações em cuidados primários e agudos em saúde consiste em uma estratégia de enfrentamento dessa

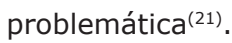

\section{Limitações do estudo}

A ausência de pesquisas no Brasil no período em que foram selecionados estudos que versam sobre a segurança do paciente e o processo do cuidar em saúde mental. Dessa forma, carece-se de novas pesquisas que explorem o assunto.

\section{Contribuições do estudo}

As contribuições deste estudo consistiram em divulgar a importância da Política de Segurança do Paciente nos serviços de saúde mental, bem como em todos os equipamentos assistenciais, que corrobore discussões e futuras pesquisas envolvendo a temática.

\section{Conclusão}

A literatura aponta que a educação permanente, a educação em saúde e a coordenação de cuidados com 
o gerenciamento dos casos são fatores essenciais para garantir a segurança da pessoa com transtorno mental.

Todavia, é necessário também atentar-se à saúde dos profissionais da área visto que o estresse e o esgotamento mental podem impactar a qualidade do atendimento $e$, consequentemente, a segurança do paciente. Dessa forma, é necessária a adoção de uma gestão sólida que valorize o reconhecimento profissional.

$O$ engajamento entre profissional e paciente auxilia na qualidade das relações interpessoais que, por sua vez, está intimamente ligada ao reconhecimento e aumento da eficácia profissional, ao mesmo tempo em que trata da individualidade do paciente de forma mais humana e não estigmatizadora.

Dentro do contexto manicomial, as pessoas ou sujeitos em sofrimento psíquico, que vivem institucionalizadas, são mais suscetíveis a eventos adversos. Diante da vulnerabilidade desta população, discutir sobre a segurança do paciente, nestes espaços, é extremamente importante na prevenção de complicações advindas de práticas manicomiais. Tal discussão favorece uma lógica de cuidado integral, que fortalece o processo de desinstitucionalização.

Apesar disso, observa-se a escassez de literatura nacional sobre o tema dentro da Psiquiatria, o que pode impactar negativamente a assistência. É necessário ampliar as pesquisas sobre a segurança do paciente psiquiátrico com vistas de reduzir a ocorrência de danos durante o cuidado dessa população.

\section{Referências}

1. Amarante PDC. Psiquiatria social e reforma psiquiátrica. 20. ed. Rio de Janeiro: FIOCRUZ; 2014

2. Yasui S. Knowing the origins of the Brazilian psychiatric reform: the French and Italian experiences. Hist Cienc Saúde-Manguinhos. [Internet]. 2011 June [cited 2018 Nov 5];18(2):585-9. Available from: http://www. scielo.br/scielo.php?script=sci_arttext\&pid=S010459702011000200016\&lng=en. doi: https://doi. org/10.1590/S0104-59702011000200016

3. Lei $n^{\circ} 10.216$, de 6 de abril de 2001 (BR). Dispõe sobre a proteção e os direitos das pessoas portadoras de transtornos mentais e redireciona o modelo assistencial em saúde mental. [Internet]. Brasília, DF; 2001. [Acesso 15 set 2020]. Disponível em: http://www.planalto.gov. br/ccivil_03/LEIS/LEIS_2001/L10216.htm\# : :text=LEI 4. Ministério da Saúde (BR). Saúde mental: o que é, doenças, tratamentos e direitos. [Internet]. Ministério da Saúde; 2019. [Acesso 4 jun 2020]. Disponível em: http:// saude.gov.br/saude-de-a-z/saude-mental

5. Ministério da Saúde (BR).Secretaria de Atenção à Saúde. DAFE. Coordenação de Saúde Mental. Reforma Psiquiátrica e política de Saúde Mental no Brasil. [Internet]. Conferência Regional de Reforma dos Serviços de Saúde Mental : 15 anos depois de Caracas. Brasília; nov 2005. [Acesso 4 jun 2020]. Disponível em: http:// bvsms.saude.gov.br/bvs/publicacoes/Relatorio15_anos_ Caracas.pdf

6. World Health Organization. The World Health Report 2001. Mental Health: New Understanding, New Hope. World Health Organization; 2001

7. Ministério da Saúde (BR). Documento de referência para o Programa Nacional de Segurança do paciente/ Ministério da Saúde; Fundação Oswaldo Cruz; Agência Nacional de Vigilância Sanitária. Brasília: Ministério da Saúde; 2014. [Acesso 3 ago 2019]. Disponível em: http://bvsms.saude.gov.br/bvs/publicacoes/documento_ referencia_programa_nacional_seguranca.pdf

8. Ministério da Saúde (BR). Gabinete do Ministro. Portaria MS/GM no 529, de 1 de abril de 2013. Institui o Programa Nacional de Segurança do Paciente (PNSP). [Internet] 2013 [Acesso 3 ago 2019]. Disponível em: http://bvsms.saude.gov.br/bvs/saudelegis/gm/2013/ prt0529_01_04_2013.html

9. Vincent C, Amalberti R. Cuidado de Saúde mais Seguro: estratégias para o cotidiano do cuidado. Rio de Janeiro; 2016. 198 p

10. Elias CSR, Silva LA, Martins MTSL, Ramos NAP, Souza MGG, Hipólito RL. When is the end? A narrative review on the termination of the school term for mentally disabled students. SMAD, Rev Eletrônica Saúde Mental Álcool Drog. jan.-abr. 2012 [cited Sep 29, 2020]; 8(1):48-53. Available from: http://www.revistas.usp.br/smad/article/ view/49594/53669

11. Cavalcanti ACD, Pereira JMV. Nursing diagnoses of patients with heart failure: an integrative review. Online Braz J Nurs. [Internet] 2014 [cited Jun 15, 2019];13(1):113-25. Available from: http://www. objnursing.uff.br/index.php/nursing/article/view/3916

12. Johnston $D$. What makes a difference to patients? Int Rev Psychiatry. 2013;25(3):319-28. doi: http://doi.org/ 10.3109/09540261.2013.782854

13. Kowalski SL, Anthony M. Nursing's evolving role in patient safety. Am J Nurs. 2017;117(2):34-48. doi: http:// doi.org/10.1097/01.NAJ.0000512274.79629.3c

14. Johnson J, Hall LH, Berzins K, Baker J, Melling K, Thompson C. Mental healthcare staff well-being and burnout: A narrative review of trends, causes, implications, and recommendations for future interventions. Int J Ment Health Nurs. 2018;27(1):20-32. doi: http://doi. org/10.1111/inm. 12416

15. Hannigan B, Simpson A, Coffey M, Barlow S, Jones A. Care Coordination as Imagined, Care Coordination as Done: Findings from a Cross-national Mental Health Systems Study. Int J Integrated Care. 2018;18(3): 12:114. doi: http://doi.org/10.5334/ijic.3978

16. Forouzan AS, Ghazinour M, Dejman M, Rafeiey $H$, Baradaran Eftekhari M, San Sebastian M. Service 
users and providers expectations of mental health care in Iran: A qualitative study. Iran J Public Health. 2013;42(10):1106-16

17. Polacek MJ, Allen DE, Damin-Moss RS, Schwartz AJA, Sharp D, Shattell M, et al. Engagement as an Element of Safe Inpatient Psychiatric Environments. J Am Psychiatr Nurs Assoc. 2015;21(3):181-90. doi: http:// doi.org/10.1177/1078390315593107

18. Grant C, Ballard ED, Olson-Madden JH. An Empowerment Approach to Family Caregiver Involvement in Suicide Prevention: Implications for Practice. Fam J. 2015;23(3):295-304. doi: http://doi. org/10.1177/1066480715572962

19. Ashmore T, Spangaro J, Mcnamara L. 'I was raped by Santa Claus': Responding to disclosures of sexual assault in mental health inpatient facilities. Int J Ment Health Nurs. 2015;24(2):139-48. doi: http://doi.org/10.1111/ inm. 12114

20. Slemon A, Jenkins E, Bungay V. Safety in psychiatric inpatient care: The impact of risk management culture on mental health nursing practice. Nurs Inquiry. 2017; 24(4):e12199. doi: http://doi.org/10.1111/nin.12199

21. Reeves E, Henshall C, Hutchinson M, Jackson D. Safety of service users with severe mental illness receiving inpatient care on medical and surgical wards: A systematic review. Int J Ment Health Nurs. 2018;27(1):46-60. doi: http://doi.org/10.1111/inm.12426

22. Sandra M, Scott BP, Heather S, Bonnie K, Joy CM. Beyond silence: protocol for a randomized parallelgroup trial comparing two approaches to workplace mental health education for healthcare employees. BMC Med Educ. 2015;15:78. doi: http://doi.org/10.1186/ s12909-015-0363-9

23. Margaret O, Conal W, Sean H. Violence within mental health services: how to enhance risk management. Risk Manag Healthc Policy. 2018:11 159-67. doi: http://doi. org/10.2147/RMHP.S131834

24. Duarte $P$, Segunda CA, Medeiros AT. Psychiatric reform in the context of anti-asylum movement struggle in Joao Pessoa - PB. [Internet]. Rio de Janeiro: Escola Nacional de Saúde Pública Sergio Arouca; 2013. Available from: https://www.arca.fiocruz.br/handle/icict/13806

25. Ministério da Saúde (BR). Política Nacional de Educação Permanente em Saúde: o que se tem produzido para o seu fortalecimento? [Internet]. Secretaria de Gestão do Trabalho e da Educação na Saúde. Brasília, DF; 2018. p. 1-78. Disponível em: https://bvsms.saude.gov.br/bvs/publicacoes/ politica_nacional_educacao_permanente_saude_ fortalecimento.pdf

26. Lima MEP de, Cortez EA, Fernandes FC, Xavier SC da M, Almeida VLA de. Permanent health education: vital strategy for safe practices. Res Soc Dev. 2020 Jun 10;9(7):721974802. doi: http://doi.org/10.33448/ rsd-v9i7.4802

\section{Contribuição dos autores}

Concepção e desenho da pesquisa: Marcos Eduardo Pereira de Lima, Elaine Antunes Cortez. Obtenção de dados: Marcos Eduardo Pereira de Lima. Análise e interpretação dos dados: Marcos Eduardo Pereira de Lima. Análise estatística: Marcos Eduardo Pereira de Lima. Redação do manuscrito: Marcos Eduardo Pereira de Lima, Elaine Antunes Cortez. Revisão crítica do manuscrito quanto ao conteúdo intelectual importante: Marcos Eduardo Pereira de Lima, Elaine Antunes Cortez, Fabíola Chaves Fernandes, Simone Costa da Matta Xavier, Viviane Lins Araújo de Almeida.

Todos os autores aprovaram a versão final do texto.

Conflito de interesse: os autores declararam que não há conflito de interesse. 